\title{
Aluminum-induced De Novo Bone Formation in the Beagle A Parathyroid Hormone-dependent Event
}

\section{D. Quarles, H. J. Gitelman, ${ }^{\star}$ and M. K. Drezner}

Departments of Medicine, Pathology, and Cell Biology, Duke University Medical Center, Durham, North Carolina 27710; and ${ }^{*}$ Department of Medicine, University of North Carolina, Chapel Hill, North Carolina 27514

\begin{abstract}
To examine the influence of osteoblast function on aluminuminduced neo-osteogenesis in the mammalian species, we compared the effects of aluminum in sham-operated and thyroparathyroidectomized (TPTX) beagles. TPTX dogs received sufficient calcium carbonate and calcitriol to maintain normal plasma calcium and calcitriol levels, but developed evidence of decreased osteoblast recruitment and activity, including diminished osteoid-covered trabecular bone surface $(3.22 \pm 0.21$ vs. $10.95 \pm 1.30 \%)$ and a decreased osteoblast number $(27.8 \pm 8.1$ vs. $139.0 \pm 26.0 / \mathrm{mm})$. Administration of aluminum (1.25 $\mathrm{mg} / \mathrm{kg}$ i.v., three times/wk) increased the serum aluminum levels in both sham $(1,087.0 \pm 276.0$ vs. $2.7 \pm 0.8 \mu \mathrm{g} / \mathrm{liter})$ and TPTX animals $(2,786.0 \pm 569.0$ vs. $3.6 \pm 0.8 \mu \mathrm{g} /$ liter $)$ above normal but did not alter the plasma calcium, creatinine, or PTH from control levels in either sham or TPTX dogs. After 8 wk of therapy, however, bone biopsies from sham-operated beagles displayed evidence of neo-osteogenesis including an increased bone volume $(47.0 \pm 1.0$ vs. $30.4 \pm 0.9 \%)$ and trabecular number $(4.1 \pm 0.2$ vs. $3.2 \pm 0.2 / \mathrm{mm})$. Much of the enhanced volume resulted from deposition of poorly mineralized woven bone $(9.9 \pm 2.7 \%)$. In contrast, biopsies from aluminum-treated TPTX animals exhibited significantly less evidence of ectopic bone formation. In this regard, bone $(35.5 \pm 1.7 \%)$ and woven tissue volume $(1.4 \pm 0.8 \%)$ as well as trabecular number $(3.3 \pm 0.1 / \mathrm{mm})$ were significantly less than those of the aluminum-treated controls. These observations illustrate that aluminum reproducibly stimulates neo-osteogenesis and induces a positive bone balance. However, this effect apparently depends on the availability of a functional osteoblast pool which, if depleted by TPTX, limits the expression of aluminum-induced new bone formation.
\end{abstract}

\section{Introduction}

Aluminum administration to adult beagles results in the unique induction of de novo bone formation and a consequent rapid accretion of bone $(1,2)$. At present, the pathophysiological basis for this aluminum-mediated neo-osteogenesis remains unknown. However, the presence in treated animals of

Portions of this work have appeared in abstract form, 1987. J. Bone Miner. Res. 2:401.

Address reprint requests to Dr. L. Darryl Quarles, Box 3036, Duke University Medical Center, Durham, NC 27710.

Received for publication 22 August 1988 and in revised form 23 November 1988.

J. Clin. Invest.

(C) The American Society for Clinical Investigation, Inc.

0021-9738/89/05/1644/07 \$2.00

Volume 83, May 1989, 1644-1650 accelerated osseous matrix synthesis evidenced by an excess of woven bone suggests that this aluminum-induced trophic response may be dependent on the presence of an increased number and/or activity of osteoblasts or their precursors. Since studies of PTH effects on replication of preosteoblasts in dogs in vivo (3) and proliferation of immature osteoblasts in vitro (4) indicate that this hormone sustains the activity per number of the osteoblast precursor pool, we designed the present study to examine whether diminished osteoblast number and/or function secondary to PTH deficiency limits aluminum action. Our data indicate that osteoblast quiescence attenuates aluminum-induced neo-osteogenesis.

\section{Methods}

Study protocol. Eight 2-yr-old male beagles, weighing $\sim 15 \mathrm{~kg}$, were randomly divided into two groups. During the study all animals were housed under identical conditions in standard kennel runs and were fed a diet containing $1.2 \%$ calcium, $0.6 \%$ phosphorus, and $2,200 \mathrm{U} / \mathrm{kg}$ (of diet) vitamin $\mathrm{D}_{3}$ (Purina Lab Chow; Ralston-Purina Co., St. Louis, MO). At the inception of the study plasma was obtained for assay of ionized calcium and immunoreactive (i) ${ }^{1}$ PTH levels. Subsequently, group 1 dogs $(n=4)$ underwent thyroparathyroidectomy (TPTX) according to previously described methods (5) as a means to reduce osteoblast number and activity. In contrast, we performed thyroidectomy only in group 2 dogs (sham; $n=4$ ). Parathyroid glands in these animals were identified visually and either left in situ or reimplanted into neck muscles if necessary. After surgery we observed that within $24 \mathrm{~h}$ TPTX animals sustained a significant reduction of plasma ionized calcium levels and the plasma iPTH concentration, confirming successful induction of parathyroid deficiency (Table I). Accordingly, we administered calcium carbonate $(5 \mathrm{~g} / \mathrm{d})$ and calcitriol $(0.125 \mu \mathrm{g} / \mathrm{d})$ supplements to these animals for the remainder of the study and thereby maintained normal plasma 1,25-dihydroxyvitamin D levels as well as normocalcemia (Table II). In addition, we treated sham and TPTX dogs with thyroxine $(500 \mu \mathrm{g} / \mathrm{d})$ to sustain normal free thyroxine $\left(\mathrm{T}_{4}\right)$ concentrations $(1.2 \pm 0.1$ and $1.1 \pm 0.1 \mathrm{ng} / \mathrm{dl}$, respectively; normal, $1.3 \pm 0.1)$.

2 mo after surgery, when sufficient time had elapsed to achieve a new steady state of bone remodeling activity (3), we obtained plasma for measurement of biochemistries and a bone biopsy from all of the dogs in each group to serve as baseline data for future studies and to confirm the presence of low bone turnover in TPTX dogs. Subsequently, we initiated treatment in both TPTX and sham beagles with aluminum chloride, $1.25 \mathrm{mg} / \mathrm{kg}$ i.v., three times per week for $8 \mathrm{wk}$. At completion of therapy we again obtained plasma for biochemical measurements and repeated bone biopsies in the treated animals.

Biochemical studies. Plasma calcium, phosphorus, creatinine, and alkaline phosphatase were measured by Auto Analyzer techniques

1. Abbreviations used in this paper: ES/BS, eroded (resorptive) surface; Fib/TV, volume of marrow fibrosis; i, immunoreactive; N.Ob, osteoblast number; Ob.S/BS, osteoblastic surface; O.Th, osteoid seam width (thickness); OV/TV, osteoid volume; $\mathrm{T}_{4}$, thyroxine; Tb.BV/TV, trabecular bone volume; TPTX, thyroparathyroidectomy; TV.W/TV, woven tissue volume. 
Table I. Plasma Ionized Calcium and PTH Concentrations before and after Sham Operation and TPTX

\begin{tabular}{clllll}
\hline & \multicolumn{2}{c}{ Sham } & & \multicolumn{2}{c}{ TPTX } \\
\cline { 2 - 3 } \cline { 5 - 6 } & Preop & Postop & & Preop & Postop \\
\hline Ionized calcium & & & & & \\
$(\mathrm{mmol} / \mathrm{liter})$ & $1.29 \pm 0.02$ & $1.30 \pm 0.02$ & $1.30 \pm 0.01$ & $0.96 \pm 0.02^{* *}$ \\
PTH $(\mathrm{pg} / \mathrm{ml})$ & $25.3 \pm 1.0$ & $25.1 \pm 1.2$ & $25.5 \pm 3.1$ & $4.9 \pm 2.6^{* \ddagger}$
\end{tabular}

* Significantly different from respective non-aluminum treated baseline controls at $P<0.05$ (paired $t$ test).

${ }^{\ddagger}$ Significantly different from respective shams at $P<0.05$ (unpaired $t$ test).

(Technicon Instruments Corp., Tarrytown, NY). The plasma ionized calcium concentration was determined with an analyzer (ICA; Radiometer Inc., Copenhagen, Denmark), and values were corrected to a $\mathrm{pH}$ of 7.4. The free $\mathrm{T}_{4}$ concentrations were assayed by RIA (Beckon, Dickinson \& Co., Orangeburg, NY) according to previously described techniques (6). The intra- and interassay coefficients of variation for the assay were 6.7 and $8.1 \%$, respectively.

We measured the plasma iPTH concentration using an RIA designed to measure the amino-terminal portion of the molecule (7). In previous studies we validated assay sensitivity by demonstrating that parathyroidectomy or induction of vitamin $\mathrm{D}$ deficiency in dogs resulted in appropriate alteration of the circulating hormone level (1).

We assayed the plasma aluminum concentration according to previously described methods (8) using a flameless atomic absorption spectrophotometer (Perkin-Elmer Corp., Norwalk, CT) with a graphite furnace. During periods of aluminum chloride treatment, plasma was obtained for assay $24 \mathrm{~h}$ after intravenous administration of the drug.

Bone studies. Transcortical bone biopsies were obtained from the anterior iliac crest of group 1 and 2 dogs under general anesthesia. We administered tetracycline $\mathrm{HCl}(15 \mathrm{mg} / \mathrm{kg}$ i.v. $)$ to each dog over a 48-h period from day 21 to day 19 before biopsy and calcein $(15 \mathrm{mg} / \mathrm{kg}$ i.v.) from day 6 to day 4 before biopsy. Bone specimens were fixed in ethanol, embedded in methyl methacrylate unstained or prestained by the methods of Villanueva (9), and sections were prepared for histomorphological examination as previously described (1). Staining for aluminum was performed by a modification of methods reported by Maloney et al. (10), while bone aluminum content was measured according to previously reported techniques (8).

Histomorphometric analysis of trabecular bone was performed with a semiautomated system (Osteoplan; Carl Zeiss, Inc., Thorn- wood, NY) as detailed elsewhere (11). The following previously defined histologic functions (7) were quantitated: $(a)$ trabecular bone volume (Tb.BV/TV); (b) osteoid volume (OV/TV); (c) woven tissue volume (TV.W/TV); $(d)$ osteoid seam width $(\mathrm{O} . \mathrm{Th}) ;(e)$ eroded (resorptive) surface (ES/BS); $(f)$ osteoblastic surface (Ob.S/BS); $(g)$ osteoblastic number (N.Ob); $(h)$ osteoid surface (OS/BS); and $(i)$ volume of marrow fibrosis (Fib/TV).

Statistical analysis. Results are expressed as the mean \pm SEM. Statistical evaluation for differences between groups was done by twotailed $t$ analysis, while that within groups by a paired $t$ test. All computations were performed with the Statgraphics software package (Statistical Graphics Corp., Inc., Princeton, NJ) on an IBM PC-AT computer.

Material. Adult beagles were obtained from Hazelton Research Animals Corp. (Cumberland, VA). Calcitriol for use in the treatment of TPTX animals was a gift from Dr. Milan Uskokovic, Hoffman-La Roche, Inc. (Nutley, NJ).

\section{Results}

Induction of diminished osteoblast number and function. 2 mo after parathyroidectomy, TPTX dogs receiving dietary supplements exhibited biochemistries comparable in most respects to those of sham-operated animals. In this regard, we observed that the plasma calcium, creatinine, calcitriol, and alkaline phosphatase levels were no different. As expected, however, the TPTX beagles did manifest a significantly reduced plasma iPTH concentration. Accordingly, their plasma phosphorus levels were increased compared with those of the sham-operated dogs (Table II).

In contrast, quantitative histomorphologic analysis of bone biopsies revealed that the TPTX dogs manifested a significant alteration in remodeling dynamics consistent with decreased osteoblast function and turnover (Fig. 1). In particular, the osteoblast number and the extent of osteoblast covered trabecular bone surfaces were significantly decreased as was the osteoid seam thickness and the osteoid surface, changes indicative of both a diminished number and activity of osteoblasts. Concordantly, the eroded surface had similarly decreased consistent with a diminished PTH stimulus (Table III).

Effects of aluminum administration. Administration of aluminum at a relatively high dose $(1.25 \mathrm{mg} / \mathrm{kg}$ i.v. 3 times/ wk) resulted in a significant elevation of the plasma aluminum concentration in both groups of treated beagles. However, in spite of receiving identical doses, TPTX animals sustained a

Table II. Plasma Biochemistries in Sham-operated and TPTX Beagles before and after Treatment

\begin{tabular}{|c|c|c|c|c|}
\hline & \multicolumn{2}{|c|}{ Sham } & \multicolumn{2}{|c|}{ TPTX } \\
\hline & Baseline & Al Rx & Baseline & Al Rx \\
\hline Total calcium $(m g / d l)$ & $9.8 \pm 0.2$ & $9.8 \pm 0.2$ & $10.2 \pm 0.1$ & $9.4 \pm 0.2^{*}$ \\
\hline Phosphorus $(m g / d l)$ & $4.23 \pm 0.16$ & $3.90 \pm 0.10$ & $4.98 \pm 0.11^{\ddagger}$ & $5.40 \pm 0.19^{\ddagger}$ \\
\hline Creatinine $(m g / d l)$ & $0.77 \pm 0.03$ & $0.77 \pm 0.04$ & $0.88 \pm 0.04$ & $0.88 \pm 0.09$ \\
\hline Alkaline phosphatase (IU/liter) & $34.8 \pm 7.7$ & $48.8 \pm 10.7$ & $29.8 \pm 7.1$ & $42.3 \pm 5.5$ \\
\hline PTH $(p g / m l)$ & $25.1 \pm 3.4$ & $22.1 \pm 2.9$ & $4.9 \pm 3.0^{\ddagger}$ & $5.3 \pm 2.9^{\ddagger}$ \\
\hline Calcitriol $(p g / m l)$ & $51.9 \pm 12.2$ & $52.7 \pm 9.8$ & $63.5 \pm 13.8$ & $43.6 \pm 4.6$ \\
\hline Free T4 $(n g / d l)$ & $1.0 \pm 0.1$ & $1.4 \pm 0.2$ & $1.5 \pm 0.2$ & $1.2 \pm 0.1$ \\
\hline Serum aluminum ( $\mu g /$ liter $)$ & $2.7 \pm 0.8$ & $1087.0 \pm 276.0^{\ddagger}$ & $3.6 \pm 0.8$ & $2786.1 \pm 569.0^{* \neq}$ \\
\hline
\end{tabular}

* Significantly different from respective non-aluminum treated baseline controls at $P<0.05$ (paired $t$ test). $\quad$ ‡ Significantly different from respective shams at $P<0.05$ (unpaired $t$ test). 

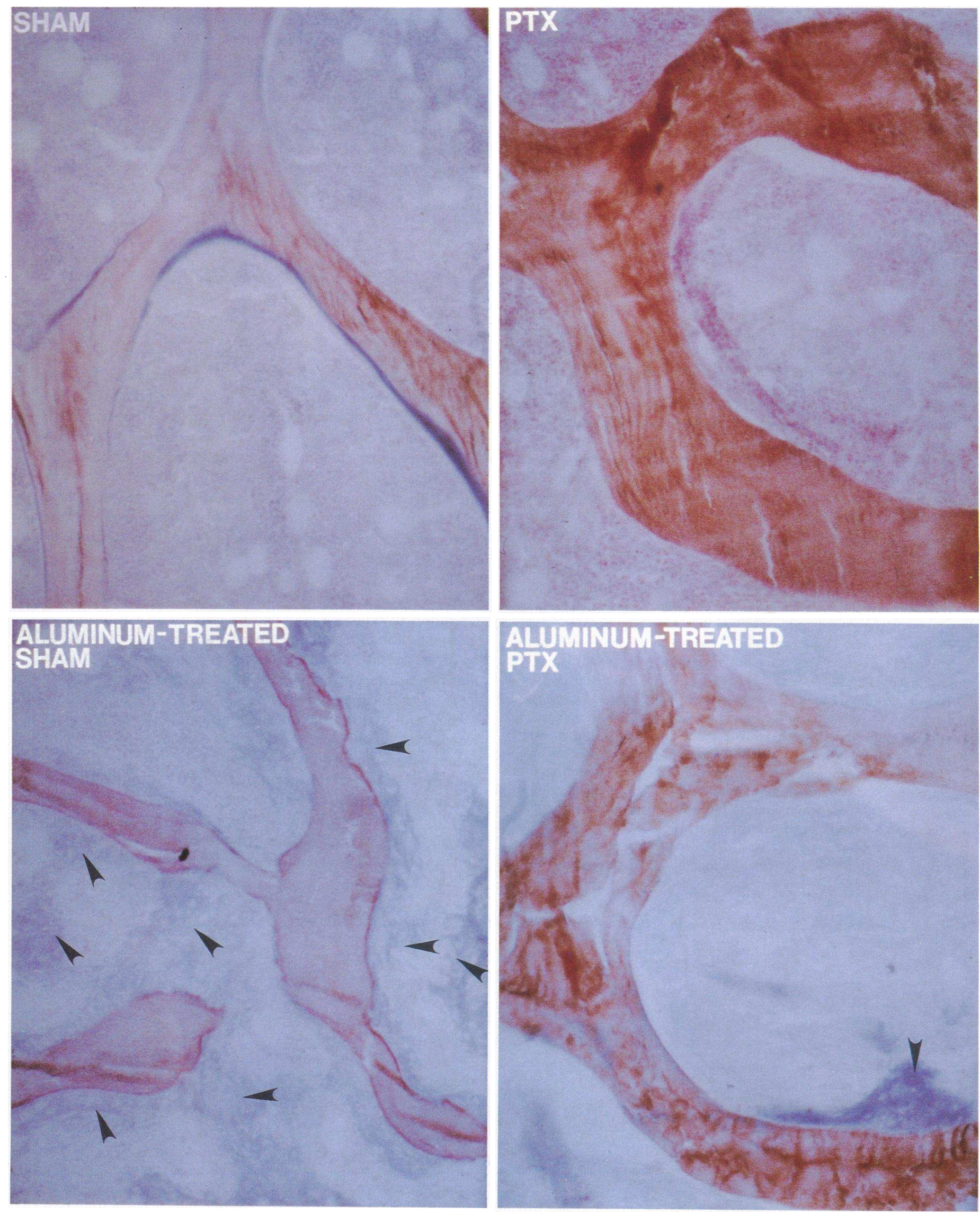

PTX 
Table III. Bone Histomorphology of Sham-operated and TPTX Beagles before and after Treatment

\begin{tabular}{|c|c|c|c|c|}
\hline & \multicolumn{2}{|c|}{ Sham } & \multicolumn{2}{|c|}{ TPTX } \\
\hline & Baseline & Al Rx & Baseline & Al Rx \\
\hline $\mathrm{BV} / \mathrm{TV}(\%)$ & $30.4 \pm 0.9$ & $47.0 \pm 1.0^{*}$ & $27.7 \pm 1.9$ & $35.1 \pm 1.7^{\ddagger}$ \\
\hline TB.N $($ No. $/ \mathrm{mm})$ & $3.2 \pm 0.2$ & $4.1 \pm 0.2^{*}$ & $2.8 \pm 0.1$ & $3.3 \pm 0.1^{* \neq}$ \\
\hline OV/TV (\%) & $0.85 \pm 0.14$ & $8.57 \pm 2.30^{*}$ & $0.13 \pm 0.03$ & $1.65 \pm 0.74^{\ddagger}$ \\
\hline TV.W/TV (\%) & 0 & $9.85 \pm 2.66^{*}$ & 0 & $1.40 \pm 0.78^{\ddagger}$ \\
\hline OS/BS (\%) & $10.95 \pm 1.30$ & $41.10 \pm 3.25^{*}$ & $3.22 \pm 0.21^{\ddagger}$ & $11.60 \pm 3.92^{\ddagger}$ \\
\hline O.Th $(\mathrm{mcm})$ & $14.4 \pm 0.5$ & $24.1 \pm 2.2^{*}$ & $9.4 \pm 0.9^{\ddagger}$ & $20.4 \pm 3.0^{*}$ \\
\hline Ob.S/BS (\%) & $4.7 \pm 1.3$ & $7.4 \pm 2.1^{*}$ & $0.9 \pm 0.2^{\ddagger}$ & $2.4 \pm 1.4$ \\
\hline $\mathrm{N} . \mathrm{Ob}(\mathrm{No} . / 10 \mathrm{~cm})$ & $139.0 \pm 26.0$ & $1582.3 \pm 734.0^{*}$ & $27.8 \pm 8.1^{\ddagger}$ & $188.4 \pm 112.5^{\ddagger}$ \\
\hline ES/BS (\%) & $3.65 \pm 0.17$ & $0.70 \pm 0.61^{*}$ & $1.30 \pm 0.55^{\ddagger}$ & $0.65 \pm 0.23$ \\
\hline $\mathrm{Fib} / \mathrm{TV}(\%)$ & 0 & $23.0 \pm 7.8^{*}$ & 0 & $39.5 \pm 22.9^{*}$ \\
\hline Bone aluminum $(\mathrm{pg} / \mathrm{ml})$ & $4.2 \pm 0.2$ & $147.7 \pm 25.1^{*}$ & $2.1 \pm 0.9^{\ddagger}$ & $62.0 \pm 11.8^{* \neq}$ \\
\hline
\end{tabular}

* Significantly different from respective non-aluminum treated baseline controls at $P<0.05$ (paired $t$ test). ${ }^{\ddagger}$ Significantly different from respective shams at $P<0.05$ (unpaired $t$ test).

significantly greater increment in the aluminum concentration than that induced in sham-operated dogs (Table I). In contrast, treatment did not differentially affect the plasma biochemistries. Indeed, after 8 wk of aluminum therapy the plasma phosphorus, creatinine, alkaline phosphatase, iPTH, and calcitriol concentrations remained unchanged from pretreatment levels of both sham-operated and TPTX beagles (Table II). Similarly, plasma calcium levels remained within the normal range in the treated animals although decreasing in the TPTX dogs receiving aluminum (Table II).

However, aluminum administration resulted in a remarkable alteration of bone histology in the sham-operated controls. Similar to our previous observations, bone biopsies from these animals exhibited abundant neo-osteogenesis (Fig. 1). Indeed, we observed new bone within the marrow space and arborizing from existing trabecular surfaces. This spatial distribution of ectopic bone resulted in a significant increase of not only the trabecular bone volume but the trabecular number as well (Table III). The presence of a marked increase in osteoblast number and deposition of the newly formed tissue largely as woven osteoid indicates that the increased bone volume resulted from an accelerated rate of synthesis. The concordant appearance of marrow fibrosis affirmed that aluminum stimulation of mesenchymal cell proliferation was probably responsible for the observed changes. In any case, the newly formed bone tissue did not completely mineralize. In fact, ongoing mineralization of woven tissue was largely limited to central sites within excrescences of woven osteoid, an activity marked by focal deposits of tetracycline (Fig. 2).

In contrast, aluminum treatment of TPTX beagles with markedly diminished osteoblast number/function and bone turnover resulted in alterations of bone histology which, although qualitatively similar, were much less pronounced. In this regard, bone biopsies exhibited significantly less ectopic bone than those of treated sham-operated dogs (Fig. 1). Indeed, the aluminum-treated TPTX animals did not significantly increase their bone volume. Moreover, their mineralized bone and woven tissue as well as the osteoblast number were less than those of aluminum-treated controls (Table III). Concordant with these disparate changes, the aluminumtreated TPTX beagles had significantly less bone aluminum accumulation than sham-operated controls, although plasma aluminum levels were maintained at a significantly elevated level. Paradoxically, the extent of marrow fibrosis remained similar to that of sham-operated animals (Table III).

\section{Discussion}

Bone formation in adult man occurs via two discrete and apparently independent processes, remodeling and neo-osteogenesis. In the former, new bone formation occurs only at sites of previous resorption and is subject to a variety of well-known hormonal/metabolic control mechanisms. In contrast, neoosteogenesis is a physiologic process that underlies primitive bone formation and fracture healing in man (12). Production of new bone as a consequence of this action depends on the independent activation/maturation of precursor cells that retain the potential of generating functional osteoblasts. Undoubtedly, a variety of factors control the recruitment of these cells during such physiologic functions. Recently, pharmacologic induction of neo-osteogenesis has also been observed. In

Figure 1. Microscopic appearance $(\times 40)$ of 5 - $\mu$ m-thick Villanueva/aluminum-stained sections of bone obtained from sham and aluminumtreated sham and TPTX and aluminum-treated TPTX beagles. Specimens from TPTX dogs, compared with those of sham-treated animals, exhibit evidence of diminished remodeling, marked by a remarkably decreased amount of blue staining osteoid covering the brown-appearing mineralized bone surfaces. In contrast, after $8 \mathrm{wk}$ of aluminum treatment, sham-operated beagles manifested abundant de novo bone histogenesis. Accordingly, sections reveal deposition of blue-staining osteoid over the trabecular envelope and within the marrow space (arrows). In contrast, specimens from aluminum-treated PTX dogs display significantly less woven osteoid (arrow) consistent with diminished new bone formation. 

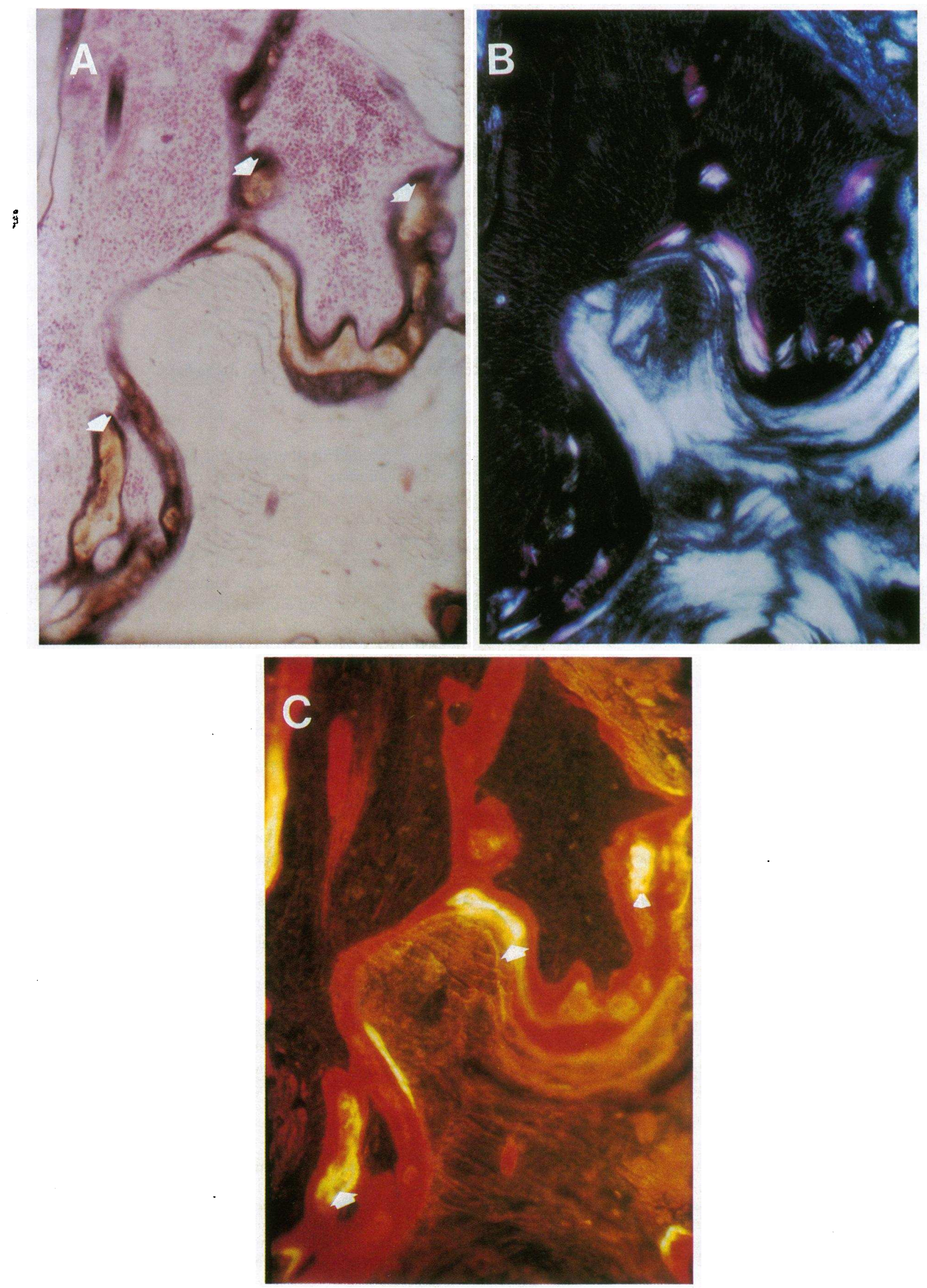

1648 L. D. Quarles, H. J. Gitelman, and M. K. Drezner 
this regard, several investigators have reported that aluminum has a trophic effect on bone matrix synthesis both in $\operatorname{dogs}(1,2)$ and rodents. These observations conclusively demonstrate that aluminum can stimulate new bone formation and substantially increase bone volume. However, virtually no information concerning the conditions under which such new bone may be induced is available. This information may be especially relevant to osteopenic disorders in which metabolic/ hormonal perturbations may alter important factors, such as the size of the preosteoblast pool, thereby limiting potentially valuable therapeutically induced neo-osteogenesis.

In this regard, the data in the present investigation provide initial evidence that an hormonally modulated decrement of osteoblast recruitment from precursor pools may significantly limit pharmacological induction of bone morphogenesis. Several observations support this conclusion. First, parathyroidectomy significantly reduced osteoblast number and activity as evidenced by histomorphometric documentation of a decrement in N.Ob, Ob.S/BS, OS/BS, and O.Th, changes probably associated with and due to a concordant reduction in the size of the preosteoblast pool. Second, aluminum administration to the TPTX beagles resulted in far less stimulation of new bone formation than apparent in similarly treated sham-operated controls. Indeed, measurements of woven tissue, bone volume, and trabecular number did not increase from baseline in the TPTX beagles. Finally, the marked reduction in neo-osteogenesis manifest in the parathyroidectomized beagles occurred concomitantly with evidence of persistently decreased osteoblast function. In this regard, bone biopsies from aluminum-treated TPTX animals exhibited an N.Ob significantly less than that of similarly managed controls. In contrast to these observations we cannot determine if aluminum has a similar trophic effect on the process of lamellar bone turnover. Indeed, we may have induced inhibition of this remodeling activity as previously reported. However, the diminished level of turnover in the TPTX animals precludes accurate assessment of such suppression.

In any case, the decreased osteoblast number and activity in the bones of the untreated and TPTX animals probably resulted from the loss of a direct PTH-mediated action to recruit cells and maintain their function. This conclusion is supported not only by previous studies $(3,4)$, but by the absence of confounding abnormalities secondary to parathyroidectomy which may indirectly influence cellular activity. In this regard, our data demonstrate that we successfully maintained plasma calcium and calcitriol concentrations within the normal range in the TPTX beagles, indicating that an alteration of calcium homeostasis did not affect the osteoblast cell pool. Similarly, a change of aluminum pharmacokinetics that would inhibit osteogenesis did not occur after parathyroidectomy. Indeed, the greater increase of plasma aluminum in TPTX beagles compared to that in sham-operated animals should have favored new bone synthesis since the circulating aluminum directly relates to the magnitude of bone histogenesis (1).
Further, the diminished bone aluminum content should have had no effect since this variable is not an important determinant of trophic events (13). Thus, the alterations in aluminum handling apparently do not underlie the altered pharmacologically induced bone formation.

While these data establish that osteoblast recruitment and/ or activity is apparently a necessary determinant for aluminum action, the mechanism by which this cellular pool is influenced and enhanced bone formation attained remains unknown. Whether the trophic response represents a direct action or is mediated through paracrine/autocrine or systemic hormonal/metabolic influences cannot be determined from this study. Nevertheless, the observed bone histogenesis is consistent with recent in vitro observations that have demonstrated the mitogenic potential of aluminum $(14,15)$. In these studies proliferation of osteoblasts derived from mouse calvariae and fibroblasts has resulted from the concerted effect of aluminum and growth factors. In this context our data suggest that a susceptible population of mesenchymal precursors display mitogenic potential in response to aluminum stimulation resulting in new bone formation and marrow fibrosis. Of note, however, the inhibition of this effect by parathyroidectomy influences only the osteoblast-mediated functions. The absence of PTH did not affect the fibroblastic response to aluminum at all. These data suggest that TPTX animals exhibit retarded development of the differentiated preosteoblast pool while retaining a population of fibroblast precursors susceptible to aluminum stimulation.

In contrast to our studies, toxic effects of aluminum, directed primarily at mature osteoblasts and the mineralization process in lamellar bone, have been reported (16-18) in studies of both man and animal models. The reasons for these disparate effects remain unclear. Species differences, the dose and/ or time of aluminum exposure, and the different experimental models (uremic vs. normal) do not readily account for the apparent variable actions of aluminum on bone. Rather, it appears likely that aluminum may exert paradoxical effects on mesenchymal precursors (including preosteoblasts) and mature osteoblasts. In this regard, aluminum effects on the precursor pools confined to the marrow space may proceed via paracrine or autocrine-mediated events, while mature osteoblasts may be influenced by the toxic potential of other aluminum-related events. Further studies will be necessary to distinguish these possibilities and the characteristics of our model system that predispose to expression of the trophic effects.

Regardless, aluminum-dependent induction of neo-osteogenesis depends on the availability of a functional osteoblast pool that, if compromised by parathyroidectomy or probably other metabolic perturbations, limits the expressed activity. Thus, as drug therapies with bone histogenetic potential are developed for osteopenic disorders, consideration must be directed at the likely attenuated effects of such treatment if the common limitation of osteoblast number and/or activity prevails.

Figure 2. Sections from the bone biopsy of a sham-operated beagle treated with aluminum for $8 \mathrm{wk}$. (A) 5- $\mu \mathrm{m}$ Villanueva stained specimen ( $\times 40)$ exhibiting tan-appearing mineralized bone covered with blue-colored osteoid in which islands of calcified tissue are apparent (arrows). $(B)$ View of the same section under polarized light which demonstrates the lamellar structure of the mineralized bone in comparison with the woven nature of the superimposed osteoid in which are included calcified tissue structures. $(C)$ The same Villanueva stained section viewed under fluorescent light, demonstrating the deposition of tetracycline in heterogenous patches (arrows) throughout the red-appearing osteoid. 


\section{Acknowledgments}

We are indebted to Ms. Jeane Raye, Ms. Amy Swartz, and Mr. Steven Castillo for excellent technical assistance.

This study was supported in part by grants from the National Institutes of Health (AR-27032 and DK-38015) and a research grant from the National Foundation (1-852). Dr. Quarles is a recipient of a Young Investigator Award (AR-37308) from the National Institutes of Health.

\section{References}

1. Quarles, L. D., H. J. Gitelman, and M. K. Drezner. 1988. Induction of de novo bone formation in the beagle. A novel effect of aluminum. J. Clin. Invest. 81:1056-1066.

2. Galceran, T. J., J. Finch, M. Bergfeld, J. W. Coburn, S. Teitelbaum, K. Martin, and E. Slatopolsky. 1987. Biological effects of aluminum on normal dogs: studies on the isolated perfused bone. Endocrinology. 121:406-413.

3. Malluche, H. H., D. Sherman, W. Meyer, E. Ritz, A. W. Norman, and S. G. Massry. 1982. Effects of long-term infusion of physiologic doses of 1-34 PTH on bone. Am. J. Physiol. 242:F197-F201.

4. Wong, G. L. 1986. Skeletal effects of parathyroid hormone. In Bone and Mineral Research. W. A. Peck, editor. 4th ed. Elsevier Science Publishing Co., Inc., New York. 103-129.

5. Malluche, H. H., M.-C. Faugere, R. M. Friedler, C. Matthews, and P. Fanti. 1987. Calcitriol, parathyroid hormone, and accumulation of aluminum in bone in dogs with renal failure. J. Clin. Invest. 79:754-761.

6. Amino, N., K. Nishi, K. Nakatani, H. Mizuta, K. Ichihara, O. Tanizawa, and K. Miyai. 1983. Effect of albumin concentration on the assay of serum free thyroxine by equilibrium radioimmunoassay with labelled thyroxine analog. Clin. Chim Acta. 29:321-325.

7. Segre, G. V. 1983. Amino-terminal radioimmunoassays from human parathyroid hormone. In Clinical Disorders of Bone and Mineral Metabolism. Elsevier Science Publishing Co., Inc., New York. 14-17.
8. Quarles, L. D., V. W. Dennis, H. J. Gitelman, J. M. Harrelson, and M. K. Drezner. 1985. Aluminum deposition at the osteoid-bone interface. An epiphenomenon of the osteomalacic state in vitamin D deficient dogs. J. Clin. Invest. 75:1441-1447.

9. Villanueva, A. R. 1974. A bone stain for osteoid seams in fresh, unembedded mineralized bone. Stain Technol. 49:1-8.

10. Maloney, N. A., S. M. Ott, A. C. Alfrey, N. L. Miller, J. W. Coburn, and D. J. Sherrard. 1982. Histological quantitation of aluminum in iliac bone from patients with renal failure. J. Lab. Clin. Med. 99:206-216.

11. Malluche, H. H., D. Sherman, R. Meyer, and S. G. Massry. 1982. A new semiautomated method for quantitative and dynamic bone histology. Calcif. Tissue Int. 34:439-448.

12. Jaworski, Z. F. G., D. B. Kimmel, and W. S. S. Jee. 1983. Cell kinetics underlying skeletal growth and bone tissue turnover. In Bone Histomorphometry: Techniques and Interpretation. R. R. Recker, editor. C. R. C. Press, Inc., Boca Raton, FL. 225-239.

13. Quarles, L. D., H. J. Gitelman, J. M. Harrelson, and M. K. Drezner. 1987. Bone aluminum: a non-essential determinant of aluminum-induced osseous disease. J. Bone Miner. Res. 1:164A. (Abstr.)

14. Lieberherr, B., B. Grosse, M. P. Witmer-Cournot, M. Hermann-Erlee, and S. Balsan. 1987. Aluminum action on mouse bone cell metabolism and response to PTH and $1,25(\mathrm{OH})_{2} \mathrm{D}_{3}$. Kidney Int. 31:736-743.

15. Smith, J. B. 1984. Aluminum ions stimulate DNA synthesis in quiescent cultures of swiss ${ }_{3} \mathrm{~T}_{3}$ cells. J. Biol. Chem. 30:31-39.

16. Hodsman, A. B., D. J. Sherrard, A. C. Alfrey, S. M. Ott, A. D. Brickman, M. L. Miller, M. A. Maloney, and J. W. Coburn. 1982. Bone aluminum and histomorphometric features of renal osteodystrophy. J. Clin. Endocrinol. Metab. 54:539-546.

17. Quarles, L. D., H. J. Gitelman, and M. K. Drezner. 1986. Aluminum: culprit or accessory in the genesis of renal osteodystrophy. Semin. Nephrol. 6:90-101.

18. Sedman, A. B., A. C. Alfrey, N. L. Miller, and W. G. Goodman. 1987. Tissue and cellular basis for impaired bone formation in aluminum-related osteomalacia in the pig. J. Clin. Invest. 79:86-92. 